\title{
Current therapeutic strategies for invasive and metastatic bladder cancer
}

This article was published in the following Dove Press journal:

OncoTargets and Therapy

II July 20 I I

Number of times this article has been viewed

\author{
Prakash Vishnu \\ Jacob Mathew \\ Winston W Tan \\ Division of Hematology Oncology, \\ Mayo Clinic, Jacksonville, FL, USA
}

Correspondence: Winston Tan Mayo Clinic, Division of Hematology Oncology, 4500 San Pablo Road, Jacksonville, FL, USA $\mathrm{Tel}+\mathrm{I} 9049536153$

Fax + I 9049536611

Email tan.winston@mayo.edu
Background: Bladder cancer is one of the most common cancers in Europe, the United States, and Northern African countries. Muscle-invasive bladder cancer is an aggressive epithelial tumor, with a high rate of early systemic dissemination. Superficial, noninvasive bladder cancer can most often be cured; a good proportion of invasive cases can also be cured by a combined modality approach of surgery, chemotherapy, and radiation. Recurrences are common and mostly manifest as metastatic disease. Those with distant metastatic disease can sometime achieve partial or complete remission with combination chemotherapy.

Recent developments: Better understanding of the biology of the disease has led to the incorporation of molecular and genetic features along with factors such as tumor grade, lymphovascular invasion, and aberrant histology, thereby allowing identification of 'favorable' and 'unfavorable' cancers which helps a more accurate informed and objective selection of patients who would benefit from neoadjuvant and adjuvant chemotherapy. Gene expression profiling has been used to find molecular signature patterns that can potentially be predictive of drug sensitivity and metastasis. Understanding the molecular pathways of invasive bladder cancer has led to clinical investigation of several targeted therapeutics such as anti-angiogenics, mTOR inhibitors, and anti-EGFR agents.

Conclusion: With improvements in the understanding of the biology of bladder cancer, clinical trials studying novel and targeted agents alone or in combination with chemotherapy have increased the armamentarium for the treatment of bladder cancer. Although the novel biomarkers and gene expression profiles have been shown to provide important predictive and prognostic information and are anticipated to be incorporated in clinical decision-making, their exact utility and relevance calls for a larger prospective validation.

Keywords: bladder cancer, chemotherapy, biologic therapy, neoadjuvant, PI3kinase/mTOR pathway

\section{Introduction}

Bladder cancer occurs mostly in men. An estimated 386,300 new cases and 150,200 deaths from bladder cancer occurred in 2008 worldwide. ${ }^{1}$ Its incidence varies widely internationally, with the highest incidence rates found in Europe, the United States, and northern African countries, while the lowest rates are found in the countries of Melanesia and middle Africa. Smoking and occupational exposures (dye, arsenic, aromatic amines, rubber or leather industries) are the major risk factors in Western countries, whereas chronic infection with Schistosoma hematobium accounts for about $50 \%$ of the total burden in developing countries. ${ }^{1,2}$ It is the fourth most common malignancy diagnosed in the US with estimates of 70,530 (52,760 men and 17,770 women) 
new cases and 14,680 (10,410 men and 4270 women $)$ deaths for the year 2010. ${ }^{1}$ The approximate 5:1 ratio of incidence to mortality reflects the frequency of superficial tumor compared with invasive and metastatic disease.

Histologically, there are three types of bladder cancer arising from the epithelium lining of the bladder. Transitional cell carcinoma, which begins in the cells lining the inner most tissue layer of the bladder, accounts for more than $90 \%$ of bladder tumors. Squamous cell carcinomas, which arise from the squamous cells of the bladder epithelium, constitute about $6 \%$ to $8 \%$ of bladder tumors and are usually associated with long-term infection or irritation of the bladder epithelium. Adenocarcinomas, which account for about $2 \%$ of bladder neoplasms, usually arise from the glandular (secretory) cells and often have urachal origin. ${ }^{3}$ The three general categories of bladder cancer - superficial, invasive and metastatic - differ in their biology, phenotype, prognosis, and management. Superficial bladder cancers, which include papillary carcinoma (Ta), carcinoma in situ 'flat tumors' (CIS) and tumors that invade subepithelial connective tissue (T1) account for about $70 \%$ of newly diagnosed urothelial bladder cancer. The initial treatment of 'nonmuscle-invasive' superficial bladder cancer is a complete cystoscopic transurethral resection of all visible tumor, usually carried out at the time of diagnosis, followed by adjuvant intravesical therapy with BCG (a live attenuated form of Mycobacterium bovis) or less commonly with mitomycin, valrubicin, gemcitabine, or thiotepa. ${ }^{4}$ Despite aggressive treatment approximately $25 \%$ of these patients with nonmuscle-invasive superficial bladder cancer develop an invasive and metastatic form of disease. ${ }^{5}$

Muscle-invasive bladder cancer is an aggressive epithelial tumor with a high rate of early systemic dissemination and poor long-term survival; almost $50 \%$ of these patients develop metastases and ultimately succumb to their disease. ${ }^{6,7}$ The most common site of metastasis of urothelial carcinoma is to the regional lymph nodes $(78 \%)$; other common metastatic sites include liver (38\%), lung (36\%), bone $(27 \%)$, adrenal gland $(21 \%)$, and intestine $(13 \%) .{ }^{8}$ In the same series, Babaian and colleagues had reported metastases to the heart, brain, kidney, spleen, pancreas, meninges, uterus, ovary, prostate, and testes in $1 \%$ to $8 \%$ of their patients. ${ }^{8}$ Although only one-third of newly diagnosed bladder cancers are advanced at presentation, another $15 \%$ to $30 \%$ of highgrade superficial tumors progress to muscle-invasive tumors usually within 5 years. ${ }^{6,7,9}$

In this article, we discuss the current multimodality strategies including systemic chemo/biologic therapies in combination with surgery and/or radiation applicable for invasive and metastatic bladder cancers, and various prognostic and predictive factors to determine therapeutic outcomes and potential for treatment-related toxicities. We performed a systematic review of peer-reviewed publications identified through searches of MEDLINE/PubMed from March 2005 to March 2011. We also included results of the relevant clinical trials presented at the annual oncology meetings (eg, Annual Meeting of American Society of Clinical Oncology [ASCO]). The ongoing Phase II and Phase III trials for first- and second-line chemotherapy for invasive and metastatic bladder cancer were searched from the US National Institute of Health's web resource, clinicaltrials.gov, which is a registry of clinical trials conducted in the US and worldwide. Keywords were used alone and with the modifiers of treatment, novel therapies, invasive and metastatic bladder cancer, and biomarkers. Bibliographies from these references were reviewed. Criteria used for study selection included study design, English language, relevance to clinicians, and validity based on the venue of publication.

\section{Staging considerations in bladder cancer}

Clinical assessment of primary tumor includes bimanual examination under anesthesia before and after endoscopic biopsy or resection and histological verification for the presence of tumor. Finding of bladder wall thickening, or a fixed mass suggest the presence of an invasive disease. Appropriate imaging studies such as a computed tomography or magnetic resonance imaging should be incorporated into clinical staging to assess the extravesical extension of the tumor and lymph node evaluation, but one should take caution for there is a potential for an overestimation or even underestimation of the stage of the tumor. The ability of these studies to determine degree of muscle invasiveness preoperatively is modest and pathologic staging is usually needed to confirm the extent of the disease. Detailed staging information is not discussed here ${ }^{10}$ in the context of this article, according to the 2010 American Joint Committee on Cancer (AJCC) cancer staging system, muscle infiltrating disease is considered T2. It is further subdivided into T2a (inner half) or T2b (outer half), but with disease still confined within the bladder. T3 lesions extend beyond muscle into the perivesical fat. T4 lesion are those extending into adjacent organs - tumors invading the prostatic stroma, vagina, uterus, or bowel are classified as T4a, while those fixed to the abdominal wall, pelvic wall, or other organs are classified as T4b. A single lymph node metastasis in the true pelvis is considered N1 disease, while multiple nodal involvement in the true pelvis 
is N2 disease, and involvement of the common iliac nodes is staged as N3. Presence of distant metastatic disease (eg, lung, liver, bones) is classified as M stage. The number of lymph nodes examined from the operative specimen and the number of positive lymph nodes have been reported to be associated with survival. ${ }^{11-13}$ In addition, the size of the largest tumor deposit and presence of extra-nodal extension may independently affect survival. ${ }^{14}$ Adequate lymph node sampling should include an average of $>12$ lymph nodes. ${ }^{10}$

\section{Prognostic and predictive markers for bladder cancer}

The most important prognostic determinants in bladder cancer are the tumor grade and stage (whether the tumor is organ-confined or nonorgan-confined). However, conventional histopathologic evaluation criteria are limited in their ability to accurately predict tumor behavior. A number of clinical and molecular characteristics are correlated with the response to chemotherapy and survival. Poor performance status and presence of visceral (eg, pulmonary, liver, skeletal) metastatic disease are correlated with decreased survival. This was demonstrated in the intergroup trial that compared cisplatin alone with methotrexate, vinblastine, doxorubicin, and cisplatin (M-VAC) in patients with metastatic disease. ${ }^{15}$ The median survival of the group with favorable features was 18.2 vs 4.4 months for the group with unfavorable features. In the long-term follow-up results of this trial, no patients with liver or bone metastases and only one patient with a Karnofsky performance status $<80$ survived 6 years. ${ }^{16}$ Subsequent reports have also confirmed the relationship between shortened survival and poor performance status or the presence of visceral metastases. ${ }^{17,18}$

Prediction tools, also known as 'nomograms', developed based on retrospective multivariate analysis to predict probability of extravesical extension or nodal metastasis at radical cystectomy, estimate the risk of recurrence and survival after cystectomy in bladder cancer and are currently available for clinical use. ${ }^{19-21}$ One such nomogram developed by International Bladder Cancer Nomogram Consortium (http:// www.mskcc.org/applications/nomograms/bladder) is based on a retrospective multivariate analysis of more than 9000 patients from twelve centers of excellence worldwide. ${ }^{20}$ This nomogram estimates probability of remaining disease free at 5 years after cystectomy based on patient age, sex, time from diagnosis to surgery, pathologic tumor stage and grade, tumor histologic subtype, and regional lymph node status. The predictive accuracy of the constructed international nomogram (concordance index, 0.75) is significantly better than standard
AJCC TNM staging (concordance index, 0.68; $P<0.001$ ) or standard pathologic subgroupings (concordance index, $0.62 ; P<0.001)$. These nomograms do not make treatment recommendations, but simply provide a means to predict an advanced stage and assess individual patient risk for disease recurrence, and survival - all key factors in deciding the need for additional treatments in the form of neoadjuvant or adjuvant therapies. These predictive tools can be accessed online at http://www.nomograms.org.

Several studies on molecular alterations as markers for prognostication have been reported with the goal of using these molecular markers of an individual tumor to help select appropriate therapy. P53 is the most widely investigated molecular marker in bladder cancer. Overexpression of P53 detected by immunohistochemistry (IHC) which infers mutation of TP53 gene has been demonstrated to be a predictor of poor survival in patients with advanced bladder cancer. ${ }^{22-24}$ In a report of 90 patients undergoing neoadjuvant $\mathrm{M}-\mathrm{VAC}$ chemotherapy, those who harbored mutant P53 were three times more likely to die from their disease than those with wild-type P53. ${ }^{25} \mathrm{Ki}-67$ index is also significantly greater in high-grade tumors and in those overexpressing P53; high Ki-67 index ( $>32 \%$ staining in IHC) is predictive of poor prognosis. ${ }^{23}$ Positive staining for pro-apoptotic markers Bax and CD40 L - is shown to predict improved survival while positive staining for anti-apoptotic marker - Bcl-2 - is correlated with poor survival. ${ }^{26,27}$ However, there have been inconsistencies in these findings which may be due to arbitrary cut-off levels for positive or negative expression based on the level of IHC staining.

Molecular profiling and proteomics can provide better indicators of tumor behavior, and may become available for routine clinical practice. ${ }^{28,29}$ A recent report from a German group $^{30}$ on the gene expression analysis of chemotherapy response modifiers multidrug resistance gene 1 (MDRl) and excision repair cross-complementing 1 (ERCC1) performed on tumor samples from patients undergoing adjuvant chemotherapy for locally advanced bladder cancer showed that expression of MDR1 and ERCC1 were independently associated with overall progression-free survival (PFS) with relative risk of 2.9 and 2.24, respectively. In another study of 57 patients with advanced bladder cancer treated with cisplatinbased regimen, the median survival was significantly longer in patients with low ERCC1 levels. ${ }^{31}$ The $M D R 1$ gene product P-glycoprotein (Pgp) is an energy-dependent efflux pump, which, among others, reduces intracellular concentrations of certain chemotherapeutic drugs including anthracyclines and vinka alkaloids, both of which are components of M-VAC 
regimen. Although cisplatin is not considered a de novo substrate of Pgp, studies have suggested an altered expression of $M D R 1$ after cisplatin administration, possibly resulting in decreased cytotoxic efficacy. ${ }^{32}$ ERCC1 gene is involved in DNA repair and may mediate resistance to alkylating agents. Recently, a 20-gene gene expression model was reported to be effective in predicting the pathological nodal status, thereby allowing selecting high-risk patients for neoadjuvant chemotherapy on the basis of risk of node-positive disease, while sparing others from toxic side-effects, and the delay to cystectomy. ${ }^{33}$

Currently, research on molecular prognostication of invasive bladder cancer is still in its infancy and the data generated from this work are primitive, but research certainly holds promise for future personalization of therapy by better understanding the biology of the disease, matching the appropriate group of patients with the right drug combination, and estimating the efficacy of those chemotherapeutic and biologic agents.

\section{Management of muscle-invasive bladder cancer}

The standard treatment approach for patients with localized muscle-invasive bladder cancer is radical cystectomy with urinary diversion. Reconstructive techniques such as ileal conduit, catheterizable pouch, and neo-bladder eliminate the need for external drainage devices in some male patients and provide improved quality of life for those who undergo radical cystectomy. Radical cystectomy requires removal of the bladder, adjacent organs, and regional lymph nodes. In men, it generally includes removal of the prostate and seminal vesicles, along with the urinary bladder, and in women, removal of the uterus, cervix, ovaries, and anterior vagina is usually performed en bloc with the bladder. Despite undergoing such 'radical' surgery, several patients are at risk of developing distant metastases and also loco-regional recurrence with second primary urothelial tumors in the renal pelvis, ureters, or urethra. Multimodality approaches in the form of neoadjuvant or adjuvant chemotherapy have been evaluated in randomized trials and are currently applied clinically to decrease relapses and increase cure rates. Result of such contemporary clinical trials in both neoadjuvant and adjuvant settings will be discussed in this section.

\section{Neoadjuvant therapy}

In patients with muscle-invasive bladder cancer, the most important treatment-related issues include the identification of those who can be cured with radical cystectomy alone, and those who, due to high risk of recurrence or metastasis, require a multimodality approach to achieve cure. For long, radical cystectomy has been considered the standard approach for patients with muscle-invasive bladder cancer. Despite a curative surgery, about half of these patients develop metastatic disease within 2 years, with a high mortality among those developing metastatic disease. ${ }^{6,34}$ Administering cisplatin-based systemic chemotherapy either before (neoadjuvantly) or after (adjuvantly) cystectomy has a potential to eradicate micrometastatic disease, and thereby improve survival in this group of patients. Hence, neoadjuvant chemotherapy followed by radical cystectomy is now considered by many as the new standard of care for this disease. The advantages of neoadjuvant therapy include delivery of chemotherapy through intact vasculature, which is often affected by surgery, and downsizing the tumor prior to cystectomy, thereby increasing complete resection with a likelihood of long-term remission and/or survival in such patients. Patients often tolerate greater dose intensity and more cycles of chemotherapy preoperatively than postoperatively. ${ }^{35}$ The disadvantage of such therapy is the delay of definitive local therapy in patients who do not respond to neoadjuvant chemotherapy and it could potentially be associated with disease progression. Results of several randomized clinical trials ${ }^{36-40}$ and a meta-analysis of all neoadjuvant studies in bladder cancer ${ }^{41}$ have favored this approach of platinum-based, multiagent, neoadjuvant chemotherapy followed by cystectomy over cystectomy alone (Table 1).

The largest neoadjuvant chemotherapy trial (BA06 30894) was conducted jointly by the Medical Research Council (MRC), the European Organization for Research and Treatment of Cancer (EORTC), and several international collaborators. ${ }^{36}$ In total, 976 patients with high-grade T2-T4a urothelial bladder cancer accrued over 5.5 years from 106 institutions were randomly assigned to three cycles of neoadjuvant cisplatin, methotrexate, and vinblastine (CMV) chemotherapy $(n=491)$ or no chemotherapy $(n=485)$, followed by institution's choice of definitive therapy with either radical cystectomy and/or radiation therapy. Of patients in the chemotherapy and no-chemotherapy groups $42 \%$ and $43 \%$, respectively, received radiation therapy alone as definitive therapy. Pathologic complete response (pCR) with neoadjuvant chemotherapy was 33\%. Overall survival (OS) at 3 years in the two groups was $55.5 \%$ vs $50 \%$, respectively, with an absolute survival benefit of $5.5 \%$ favoring the chemotherapy group. However, the prespecified statistical aim to detect an absolute survival improvement of $10 \%$ (from $50 \%$ to $60 \%$ ) was not met. The most recent update, ${ }^{42}$ 
Table I Results of randomized clinical trials evaluating neoadjuvant chemotherapy for muscle-invasive bladder cancer

\begin{tabular}{|c|c|c|c|c|c|}
\hline Study & $\mathbf{N}$ & Regimen & Comparator & PCR & OS \\
\hline EORTC/MRC 36 & 976 & $\begin{array}{l}\text { CMV ( } 3 \text { cycles) followed by } \\
\text { cystectomy or RT ( } n=491)\end{array}$ & $\begin{array}{l}\text { Cystectomy or RT } \\
(\mathrm{n}=485)\end{array}$ & $33 \%$ & $5.5 \%$ in favor of CMV \\
\hline INT 0080/SWOG $8710^{38}$ & 317 & $\begin{array}{l}\text { M-VAC ( } 3 \text { cycles) followed } \\
\text { by cystectomy }(n=154)\end{array}$ & $\begin{array}{l}\text { Cystectomy } \\
(\mathrm{n}=153)\end{array}$ & $38 \%$ & $\begin{array}{l}\text { Trend in benefit with } \\
M-V A C(P=0.06)\end{array}$ \\
\hline Nordic ${ }^{39}$ & 325 & $\begin{array}{l}\text { CA }(2 \text { cycles) followed by } \\
\text { cystectomy or RT }(n=|5|)\end{array}$ & $\begin{array}{l}\text { Cystectomy or RT } \\
(\mathrm{n}=160)\end{array}$ & NR & No difference \\
\hline Nordic $2^{37}$ & 317 & $\begin{array}{l}\text { CM ( } 3 \text { cycles) followed } \\
\text { by cystectomy }(n=155)\end{array}$ & $\begin{array}{l}\text { Cystectomy } \\
(\mathrm{n}=154)\end{array}$ & $26.4 \%$ & No difference \\
\hline Italy $\left(\right.$ GISTV) ${ }^{40}$ & $17 \mid$ & $\begin{array}{l}\text { M-VEC ( } 3 \text { cycles) followed } \\
\text { by cystectomy }(n=82)\end{array}$ & $\begin{array}{l}\text { Cystectomy } \\
(\mathrm{n}=7 \mathrm{I})\end{array}$ & $28 \%$ & No difference \\
\hline
\end{tabular}

Abbreviations: $\mathrm{PCR}$, pathologic complete response; OS, overall survival; CMV, cisplatin + methotrexate + vinblastine; M-VAC, methotrexate + vinblastine + adriamycin + cisplatin; NR, not reported; CA, cisplatin + adriamycin; CM, cisplatin + methotrexate; M-VEC, methotrexate + vinblastine + epirubicin + cisplatin; SWOG, South West Oncology Group; RT, radiotherapy.

after 8 years of follow-up, showed a statistically significant $16 \%$ reduction in the risk for death in patients who received neoadjuvant CMV prior to radiotherapy and/or cystectomy; this corresponds to an increase in 3-year survival from 50\% to $56 \%$, an increase in 10 -year survival from $30 \%$ to $36 \%$, and an increase in median survival time of 7 months (from 37 to 44 months) in CMV-treated patients compared with those treated with local therapy only.

In a US Intergroup trial (INT 0080), ${ }^{38} 307$ of the 317 enrolled patients with T2-T4a urothelial bladder cancer were randomized to three cycles of neoadjuvant M-VAC $(\mathrm{n}=154)$ or no chemotherapy $(n=153)$ followed by cystectomy. The study took almost 13 years to complete accrual. pCR with neoadjuvant M-VAC chemotherapy was 38\%. Median follow-up was 8.7 years. Patients who received M-VAC showed a trend towards improvement in median OS (77 vs 46 months, $P=0.06$ ). A subsequent retrospective analysis showed that after adjustment for pathologic factors and neoadjuvant chemotherapy use, an optimal cystectomy and thorough pelvic node dissection, defined as negative resection margins and at least 10 lymph nodes in the surgical specimen, was associated with longer survival ( $>80 \%$ at 5 years). ${ }^{43}$ Another recent secondary analysis of this study showed that presence of squamous or glandular differentiation in locally advanced urothelial bladder cancer does not confer resistance to M-VAC, and in fact, may be an indication for the use of neoadjuvant chemotherapy before radical cystectomy. ${ }^{44}$

Randomized clinical trials (Nordic ${ }^{37,39}$ and GISTV, ${ }^{40}$ Table 1) did not demonstrate survival difference using neoadjuvant chemotherapy.

Majority of the randomized clinical trials have not demonstrated a survival benefit with the addition of neoadjuvant chemotherapy. Inadequate sample size, suboptimal chemotherapy, premature closure, and/or inadequate follow-up have all been attributed to these negative results. Hence, meta-analyses have been performed to interpret these data. An update of a systematic review and meta-analysis of clinical trials of neoadjuvant chemotherapy in invasive bladder cancer was published by Advanced Bladder Cancer metaanalysis collaboration. ${ }^{41}$ In patients who received cisplatinbased combination chemotherapy prior to cystectomy, a 5\% absolute benefit improving survival from $45 \%$ to $50 \%$ at 5 years $(P=0.003)$ was observed. No information was reported about the quality of life and toxicities from various chemotherapeutic regimens used. Most patients from the EORTC/MRC, INT 0080, and Nordic studies were young, with a median age of 63 to 65 years with excellent performance status and good renal function; hence, there remains a question as to whether these results can be applied to most of the elderly patients who form the major proportion of the bladder cancer population.

Regimens such as gemcitabine plus cisplatin (GC), which in metastatic setting is shown to be less toxic and achieves similar response rates and survival, ${ }^{45}$ has not been tested prospectively in the neoadjuvant setting. A recent singleinstitution retrospective study by Dash et $\mathrm{al}^{46}$ showed a pCR of $26 \%$ with GC which is comparable to other cisplatin-based regimens. A combination of a taxane, nab-paclitaxel, along with gemcitabine and carboplatin in a neoadjuvant setting was recently reported. ${ }^{47}$ In this Phase II trial, 27 eligible patients with T2-T4, N0, or any T, N1-3 bladder cancer were treated with three cycles of nab-paclitaxel along with gemcitabine and carboplatin, followed by cystectomy. Of the 27 patients, 25 completed all three cycles. Grade 3-4 neutropenia was seen in all patients. pCR, the primary endpoint, was seen in $30 \%$ of the patients with $25 \%$ demonstrating CIS. This combination appears to be an active regimen and could be of potential benefit in patients who are not 
candidates for cisplatin. Blick et $\mathrm{al}^{48}$ reported a retrospective study of 80 patients who underwent accelerated M-VAC therapy administered at 2-week intervals with granulocyte colony-stimulating factor (G-CSF) support in an attempt to minimize delay to definitive therapy and improve efficacy of neoadjuvant chemotherapy. All planned cycles of chemotherapy were completed by $84 \%$ of patients and median duration of chemotherapy was 34 days. All 80 patients received their planned definitive therapy (cystectomy in 60 patients; radiotherapy in 20 patients). pCR was seen in $43 \%$ of patients treated with surgery with an objective radiological response in $75 \%$ of patients. There were no treatment-related deaths, and incidence of grade $\geq 3$ toxicities was $11 \%$. Accelerated M-VAC appears to be a safe and well-tolerated regimen that needs to be prospectively evaluated. Although these newer regimens are promising, there are no data yet from well-powered randomized trials supporting their use. For those patients with inter-current illnesses that prohibit use of M-VAC, GC may constitute a reasonable alternative. Several clinical trials are evaluating biologic agents along with chemotherapeutic combinations in a neoadjuvant setting as listed in Table 2.

Addition of radiation therapy to chemotherapy in the neoadjuvant setting has been investigated in randomized studies with equivocal results; hence this approach is not considered a standard-of-care. The results of these trials will not be discussed here but are available for review elsewhere. ${ }^{49-52}$

The primary goal of muscle-invasive bladder cancer treatment is cure, and bladder preservation is a secondary consideration. Organ-sparing approaches are considered as an alternative, particularly in frail and very elderly patients and those with significant medical co-morbidities or those who will not accept the side-effects and risks associated with surgery. Avoidance of radical cystectomy as a reasonable approach in those patients who have a complete response to neoadjuvant therapy has been investigated in a few clinical trials. Herr et $\mathrm{al}^{53}$ have reported a nonrandomized study with 111 patients with T2-3, N0, M0 urothelial cancer who received neoadjuvant $\mathrm{M}-\mathrm{VAC}$ chemotherapy. Forty-three of the 60 patients who achieved complete clinical response (cT0) underwent bladder-sparing surgery (transurethral resection of bladder tumor [TURBT] alone in 28 patients; partial cystectomy in 15 patients) while 17 underwent radical cystectomy. At 10 years, 32 of the 43 patients (75\%) who underwent bladder-sparing surgery were alive. These results were similar to the group who underwent radical cystectomy (65\% survival at 10 years). However the bladder remained at risk for new invasive tumors (24 patients, 56\% relapse), most requiring salvage cystectomy. In a similar study reported by Sternberg et al, ${ }^{54} 104$ patients with T2-T4a urothelial cancer who received neoadjuvant therapy with M-VAC, were followed by bladder-sparing surgery in 65 patients (TURBT alone in 52 patients; partial cystectomy in 13 patients) while 39 patients had radical cystectomy based on the degree of response to chemotherapy. The estimated 5-year survival in the group undergoing bladder-sparing surgery was $67 \%$ compared with $46 \%$ in group who underwent radical cystectomy. However, a more recent Phase II clinical trial reported by deVere et $\mathrm{al}^{55}$ showed that though the complete clinical response (cT0) by TURBT following neoadjuvant therapy (with gemcitabine, carboplatin, and paclitaxel) was $46 \%$, there was an unacceptably high rate $(60 \%)$ of persistent

Table 2 Active clinical trials evaluating neoadjuvant chemotherapy for muscle invasive bladder cancer ${ }^{100}$

\begin{tabular}{|c|c|c|c|c|}
\hline Study identifier & Phase & Study drug & Start date & Primary endpoint \\
\hline NCT00585689 & II & gemcitabine + carboplatin + nab-paclitaxel & December 2007 & $\mathrm{pCR}$ \\
\hline NCT0070664l & Pilot (0) & dasatinib & June 2008 & Feasibility \\
\hline NCT0I 245660 & Pilot (0) & lapatinib & November 2010 & Effect on EGF pathway \\
\hline NCT008470I5 & II & gemcitabine + cisplatin + sunitinib & February 2009 & $\mathrm{PCR}$, safety \\
\hline NCT00506I55 & II & M-VAC + bevacizumab & July 2007 & $\mathrm{PCR}$ \\
\hline NCT0I 222676 & II & gemcitabine + cisplatin + sorafenib & October 2010 & $\mathrm{pCR}$ \\
\hline NCT0I03I420 & II & dose-dense M-VAC & December 2009 & $\mathrm{pCR}$ \\
\hline NCT0I094496 (N-ABLE) & II & $\begin{array}{l}\text { gemcitabine }+ \text { cisplatin } \pm \text { CDX- } 1307 \\
\text { vaccine (for tumors expressing } \beta \text {-hcg) }\end{array}$ & March 2010 & RFS \\
\hline NCT00268450 & II & $\begin{array}{l}\text { neoadjuvant gemcitabine }+ \text { cisplatin }+ \\
\text { bevacizumab, followed by surgery and } \\
\text { adjuvant bevacizumab and paclitaxel }\end{array}$ & December 2005 & $\mathrm{PCR}$ \\
\hline NCT00749892 & II & erlotinib & September 2008 & $\mathrm{pCR}$ \\
\hline NCT00I36I75 & II & gemcitabine + carboplatin + paclitaxel & August 2005 & $\mathrm{PCR}$ \\
\hline NCT0I093066 & II & M-VAC follwed by optimal TURBT & April 2010 & BPR \\
\hline
\end{tabular}

Abbreviations: pCR, pathologic complete response; EGF, epithelial growth factor; M-VAC, methotrexate + vinblastine + adriamycin + cisplatin; RFS, recurrence-free survival; BPR, bladder preservation rate; TURBT, transurethral resection of bladder tumor. 
cancer at cystectomy in patients presumed to have pT0 status. The authors concluded that patients completing neoadjuvant chemotherapy should strongly consider definitive local therapy regardless of post-chemotherapy cT0 status. Based on these studies, one can infer that a considerable number of patients whose invasive tumors are significantly downsized with combination chemotherapy may be curable by conservative surgery such as partial cystectomy, rather than a radical cystectomy; however, downsizing with neoadjuvant therapy does not necessarily ensure complete local control of disease, especially with a high risk of metachronous bladder cancer in these patients.

\section{Adjuvant therapy}

Unlike neoadjuvant treatment, adjuvant chemotherapy can be tailored according to the pathologic staging prior to administration of systemic therapy, thereby limiting toxicity associated with such treatment and also avoiding any delay in potentially curative surgery in those patients whose tumor is not responsive to cytoreductive chemotherapy. The availability of adequate tissue for analysis of molecular prognostic and predictive markers may be another advantage. The disadvantage of adjuvant therapy is that there could be delay in initiating systemic therapy for occult metastatic disease while treating the primary focus; in some surgically debilitated and elderly patients, it can be very challenging and sometimes may not be possible to administer adequate systemic chemotherapy following cystectomy.

Similar to neoadjuvant setting, there are several randomized clinical trials reported in the adjuvant setting which have conflicting results and with caveats such as inadequate sample size, flawed clinical trial design, and poor accrual leading to early termination. The older trials have been reviewed elsewhere. ${ }^{56} \mathrm{~A}$ systematic review and meta-analysis of individual patient data from those trials was published in 2005. ${ }^{57}$ The results, based on 491 patients from six trials, representing $90 \%$ of all patients randomized in cisplatin-based combination chemotherapy trials and $66 \%$ of patients from all eligible trials, suggested a $25 \%$ relative reduction in the risk of death for chemotherapy compared with that on control, with an overall hazard ratio for survival of $0.75(P=0.019)$. It concluded that there was insufficient evidence on which to reliably base treatment decisions. The contemporary cooperative trials will be reviewed in this section.

In an Italian multicenter randomized Phase III trial, ${ }^{58}$ patients with pT2G3, pT3-4, N0-2 transitional cell bladder carcinoma, after radical cystectomy, were assigned to four cycles of GC or observation followed by same chemotherapy at progression. Only 194 patients were enrolled (32\% of the target) and the trial was stopped due to poor accrual. At a median follow-up of 32.5 months, relapses were similar in both groups ( $43 \%$ vs $45 \%$ ) with no difference in disease-free survival (DFS). The 3-year OS was $67 \%$ for the chemotherapy arm and $48 \%$ for the observation arm and the 3-year DFS was $47 \%$ and $35 \%$, respectively, suggesting no improvement in OS or DFS with adjuvant GC in these patients.

In a clinical trial conducted by the Southwest Oncology Group, ${ }^{59} 499$ patients post-radical cystectomy for urothelial cancer with pT1-T2, N0 disease were assessed for P53 expression. Those positive for P53 expression with $\geq 10 \%$ nuclear reactivity by IHC were randomly assigned to observation vs three cycles of adjuvant M-VAC. Primary endpoint was recurrence-free survival. The trial was terminated after a planned interim analysis due to futility. Among the 114 patients with P53-positive tumors who were randomized to observation or adjuvant chemotherapy, there were no differences in time to recurrence or OS. In the entire cohort, the study did not confirm the prognostic value of P53 expression by IHC for either recurrence or OS.

In the randomized Phase III Spanish Oncology Genitourinary Group trial 99/01, ${ }^{60}$ patients with high-risk muscle-invasive bladder cancer (pT3-4 and/or node-positive disease) were assigned to four courses of chemotherapy with paclitaxel, gemcitabine, and cisplatin combination or observation. The primary objective was OS. The study was opened in July 2000 and prematurely closed in July 2007 due to poor recruitment, with 142 patients randomized (74 to observation and 68 to chemotherapy). At a median follow-up of 51 months, there was a statistically significant increase in OS with chemotherapy compared with observation. Five-year OS was $60 \%$ vs $31 \%$ respectively. Secondary endpoints such as DFS, time-to-progression (TTP) and disease-specific survival were also superior in the chemotherapy arm. Importantly, this abstract reports a post-hoc review of a study that was closed early to accrual, and further follow-up and peer review will be required before it can be viewed as definitive.

A large Phase III trial by EORTC (protocol \#30994) evaluating observation vs adjuvant chemotherapy with one of the three chemotherapy regimens (GC, M-VAC, or high-dose M-VAC) in high risk bladder cancer (pT3-4 and/or nodepositive disease) was also prematurely closed in August 2008 due to poor accrual after enrolment of 278 patients; another large 800-patient trial by the Cancer and Leukemia Group B evaluating role of high-dose intensity chemotherapy vs standard chemotherapy in the adjuvant setting also suffered from 
poor accrual that led to its early closure. Results of these trials are currently not available.

Based on the older clinical trials, meta-analysis, and the contemporary clinical trials in the adjuvant setting, there appears to be no clear evidence for the role of adjuvant chemotherapy in locally advanced bladder cancer. Patients are encouraged to participate in such clinical trials whenever possible. In patients with $\mathrm{pT} 2$, N0 urothelial bladder cancer, following cystectomy with observation seems to be a rational approach while for those patients with pT3-4 and/or nodepositive disease, following cystectomy with four cycles of chemotherapy with M-VAC or GC appears reasonable since these regimens have shown significant activity in metastatic setting.

\section{Management of metastatic bladder cancer}

First-line therapy

The standard approach for patients with inoperable locally advanced or metastatic disease is systemic chemotherapy. Urothelial bladder cancer is highly responsive to cisplatinbased chemotherapy; however, the median survival even with aggressive chemotherapy is only about 15 months. Several chemotherapeutic drugs such as cisplatin, methotrexate, adriamycin, ifosfamide, docetaxel, and gemcitabine have shown to have single-agent activity in either first-line or subsequent therapy of metastatic bladder cancer, but with low overall response rates (ORR) and short duration of responses. ${ }^{61-66}$ This led to development of cisplatin-based combination regimens.

In a randomized trial of 108 patients, comparing cisplatin with cisplatin plus methotrexate, ${ }^{67}$ the combination demonstrated a response rate of $45 \%$ vs $31 \%$ compared with single-agent cisplatin, which was not significantly different. There was an improved TTP but no difference in survival. In a 58-patient cohort with metastatic transitional cell carcinoma, combination of cisplatin, methotrexate, and vinblastine showed an ORR of $56 \%$ with a complete response rate (CR) of $28 \%$. Patients who had achieved CR showed a prolonged DFS of 11 months.

M-VAC regimen in a nonrandomized clinical trial ${ }^{68}$ of 133 patients with advanced urothelial tract cancer showed tumor regression in about $72 \%$ of cases and $36 \%$ of those achieved CR; 3-year survival was 55\% among patients who had a CR. Further, in a prospective randomized international cooperative group trial, M-VAC was compared with single-agent cisplatin. ${ }^{15}$ Patients (269) were assigned to M-VAC or cisplatin, cycles repeated every 28 days until tumor progression or a maximum of six cycles. M-VAC regimen was associated with a greater toxicity, particularly leukopenia, mucositis, neutropenic fever, and drug-related mortality. Response rates were superior in the M-VAC arm compared with the single-agent cisplatin arm (39\% vs $12 \%)$ PFS (10.0 vs 4.3 months) and OS (12.5 vs 8.2 months) were significantly greater for the combined therapy arm. In another randomized trial with 110 patients, ${ }^{69} \mathrm{M}-\mathrm{VAC}$ was compared with a regimen consisting of cisplatin, cyclophosphamide, and doxorubin (CISCA); M-VAC arm showed significantly higher response rate $(65 \%$ vs $46 \%)$ and median survival (48 vs 36 weeks) compared with CISCA. In attempts to translate response rates to improved survival rates, high-dose intensity M-VAC was evaluated in an EORTC Phase III clinical trial (protocol \#30924) ${ }^{70}$ with a recent 7 -year update of the results. ${ }^{71}$ Patients (263) were randomly assigned to high-dose M-VAC given at 2-week intervals with growth factor support or to standard M-VAC given every 4 weeks. ORR (63\% vs 50\%), CR ( $21 \%$ vs $9 \%$ ), and PFS (9.1 vs 8.2 months) were improved but there was no difference in OS, which was the primary endpoint (15.5 vs 14.1 months). In the subsequent update with more than 7 years of follow-up, high dose M-VAC showed a borderline statistically significant relative reduction in the risk of death at 5 years $(21.8 \%$ vs $13.5 \%$; hazard ratio $=0.76)$ compared with M-VAC. Toxicity is a major consideration with M-VAC particularly myelosupression, neutropenic fevers, sepsis, and mucositis, with significant toxicity-related deaths reported in most clinical trials evaluating M-VAC. High dose M-VAC is considered standard of care at some centers, but not all.

In Phase II clinical trials, gemcitabine in combination with cisplatin have shown response rates of about $50 \%$ with a median survival of around 14 months. ${ }^{72,73}$ Based on these results, this combination was evaluated in a randomized Phase III trial of 405 patients, comparing it with M-VAC.45 Chemotherapy was administered every 4 weeks for a maximum of six cycles. More patients in the GC arm completed the planned six cycles of therapy with fewer dose adjustments and significantly fewer patients with neutropenia and related complications, and toxicity-related deaths. The ORR (49\% vs $46 \%$ ), TTP (7.4 vs 7.4 months), and median survival (13.8 vs 14.8 months) were similar in both groups. This study demonstrated that GC had a better safety profile and tolerability while providing similar survival benefit compared with M-VAC. An updated analysis showed similar 5-year survival rates between the two regimens. ${ }^{17}$ Based on its similar efficacy and lower toxicity, GC rather than M-VAC 
is considered by many to be the standard first-line regimen for patients with advanced urothelial bladder cancer.

Addition of paclitaxel to GC was evaluated in a Phase III clinical trial by EORTC (protocol \#30987) ${ }^{74}$ which enrolled 627 chemotherapy-naïve patients with advanced urothelial carcinoma, $81 \%$ of whom had primary bladder tumors. Chemotherapy was administered for a maximum of six cycles. Both regimens were well tolerated overall. Results showed that the triplet combination had a higher rate of ORR (57\% vs $46 \%$ ) and CR (15\% vs 10\%); though the survival was 3 months longer (15.7 vs 12.8 months) in the 3 -drug arm, it was not statistically different from GC.

Combination of docetaxel and cisplatin (DC) has been compared with M-VAC in a multicenter Phase III clinical trial by the Hellenic Co-operative Oncology group. ${ }^{75}$ Patients (220) were randomly assigned to M-VAC every 4 weeks vs docetaxel plus cisplatin every 3 weeks. Treatment with M-VAC resulted in significantly superior RR (54.2\% vs 37.4\%), median TTP (9.4 vs 6.1 months), and median survival (14.2 vs 9.3 months), suggesting that M-VAC was superior to DC. Toxicity of M-VAC was considerably lower than that previously reported for M-VAC administered without G-CSF.

A Phase II trial ${ }^{76}$ comparing gemcitabine plus carboplatin with GC and an Eastern co-operative Oncology group (ECOG) Phase III trial ${ }^{77}$ comparing M-VAC with carboplatin plus paclitaxel have evaluated carboplatin-based regimens, but have not established the equivalence of carboplatin to cisplatin. A recent EORTC trial ${ }^{78}$ (protocol \#30986) has compared gemcitabine plus carboplatin with methotrexate, carboplatin plus vinblastine (M-CAVI) in patients with advanced urothelial cancer who are 'unfit' to receive cisplatin due to renal dysfunction or other medical comorbidities. With 178 patients enrolled, this study showed an ORR of $42 \%$ for gemcitabine plus carboplatin and $30 \%$ for that M-CAVI, suggesting that both these regimens are active in this group of cisplatin 'unfit' patients. Age alone is not a contraindication for cisplatin use.

Two Phase II studies have reported antitumor efficacy of the combination of gemcitabine with pemetrexed, a folate antimetabolite, in patients with untreated metastatic urothelial cancer, demonstrating a moderate antitumor activity at the expense of significant myelosuppression. In the ECOG study (E4802) ${ }^{79}$ with a cohort of 46 patients treated for a maximum of six cycles, the ORR was $31.8 \%$; median TTP was 5.8 months with a median OS of 13.4 months. The most common grade $\geq 3$ toxicity was neutropenia ( $75 \%$ ) with $11 \%$ febrile neutropenia. In an earlier study of 64 patients, ${ }^{80}$ the reported ORR was $20 \%$ in the intention-to-treat population ( $28 \%$ among the 47 patients evaluable for response); median OS was 10.3 months. Significant grade $\geq 3$ toxicity included neutropenia (38\%) with febrile neutropenia (17\%) and anemia $(19 \%)$.

Eribulin, currently approved by the US Food and Drug Administration (FDA) to treat patients with metastatic breast cancer based on the results of a Phase III EMBRACE trial, ${ }^{81}$ is a synthetic analog of halichondrin $\mathrm{B}$ and a potent inhibitor of microtubule dynamics. Preliminary results from an ongoing Phase II trial evaluating eribulin in patients with urothelial cancer with no prior cytotoxic therapy for advanced disease (neo/adjuvant therapy allowed) was recently reported. ${ }^{82}$ Results of the 37 evaluable patients demonstrated an ORR of $38 \%$ and a RR of $34 \%$ in patients who had received prior neo/adjuvant therapy. At a median follow-up of 19.8 months, the PFS was 3.9 months and a median OS of 9.4 months, suggesting promising activity of eribulin in this group of patients. The most common grade $\geq 3$ toxicity reported was neutropenia (54\%). Its safety and efficacy in combination with GC is currently being evaluated in a Phase I/II study (Table 2).

With a better understanding of tumor biology including a few upregulated/dysregulated signaling pathways (Figure 1) in urothelial cancer, ${ }^{83}$ several agents that act against specific targets among these signaling pathways, particularly vascular endothelial growth factor receptor (VEGFR) (eg, bevacizumab), epithelial growth factor receptor (EGFR) (eg, cetuximab), and mammalian target of rapamycin (mTOR) (eg, everolimus) are currently being tested in first-line therapy in combination with cytotoxic chemotherapy for patients with advanced bladder cancers, some of the agents showing promising results (Table 3; Figure 1).

Bevacizumab has been studied in combination with GC as first-line therapy for metastatic urothelial carcinoma in a Phase II trial by the Hoosier Oncology group ${ }^{84}$ (GU 04-75). In this single-arm study, 43 patients received GC along with bevacizumab $15 \mathrm{mg} / \mathrm{kg}$ every 3 weeks. Known antiangiogenic treatment-related toxicities (bleeding, thromboembolism) were common. The ORR was $72 \%$ with a CR of $21 \%$, another $16 \%$ having stable disease. At a median follow-up of 27.2 months, PFS was 8.2 months with an OS of 20.4 months, suggesting that the combination of GC and bevacizumab is an active first-line regimen in metastatic bladder cancer. This is now being tested in a large Phase III trial (Table 3 ).

Human epidermal growth factor receptor-2 (Her2/neu, c-erbB2) expression and efficacy of combining trastuzumab (a humanized monoclonal antibody that binds to Her2/neu) 


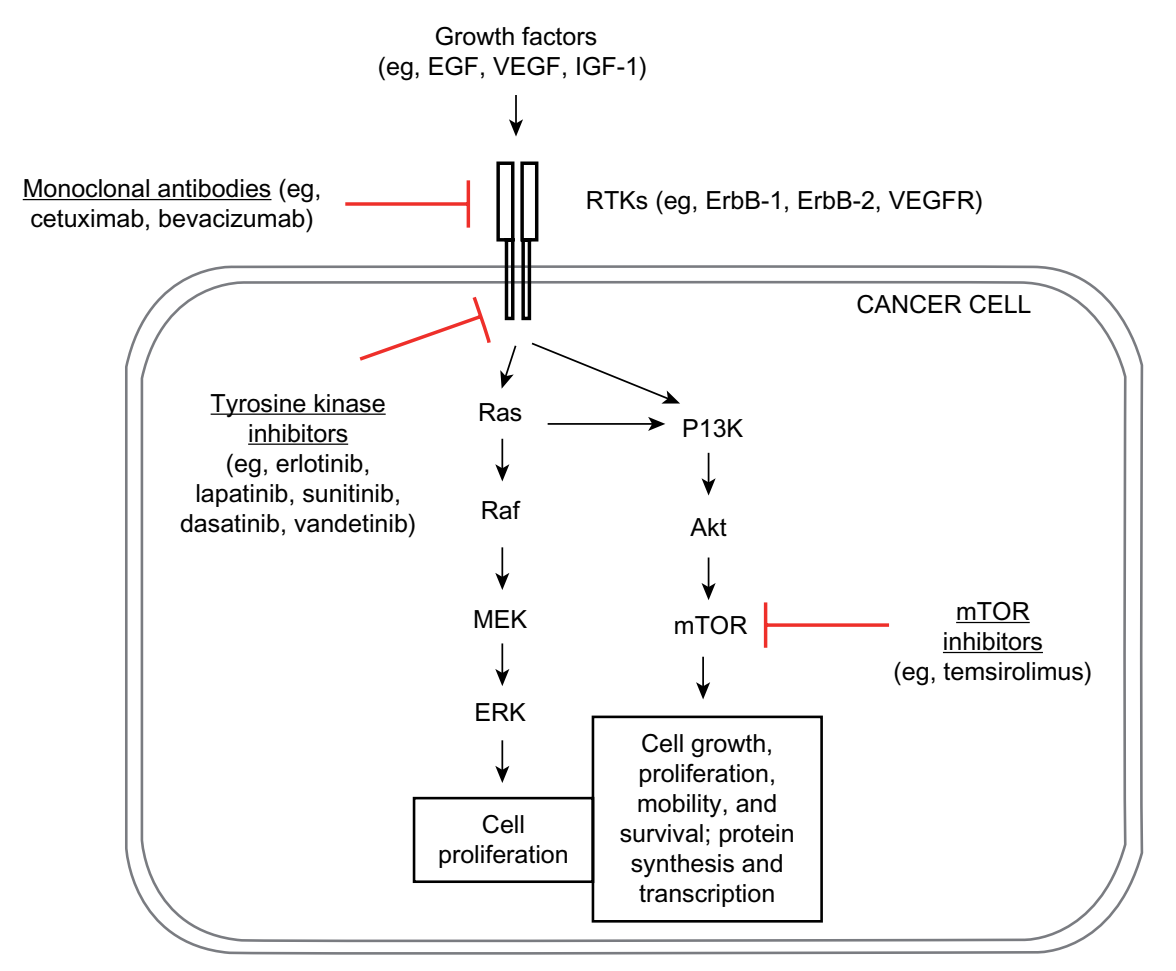

Figure I Dysregulated signaling pathways and targeted therapy in bladder cancer.

Abbreviations: EGF, Epithelial Growth Factor; VEGF, Vascular Endothelial Growth Factor; IGF-I, Insulin-like Growth Factor-I; RTK, Receptor Tyrosine Kinase; mTOR, mammalian Target of Rapamycin.

with first-line therapy of recurrent and/or metastatic Her2/neupositive urothelial cancers was reported in a Phase II study. ${ }^{85}$ Expression of Her $2 /$ neu in urothelial cancers can be variable, ranging from $8.5 \%$ to $81 \%$, and in this study $52.3 \%$ of the tumors were positive (57 of the 109 screened cases).
Her-2/neu-positive patients had more metastatic sites and visceral metastasis than did Her-2/neu-negative patients. Forty-four of the $57 \mathrm{Her}-2 /$ neu-positive patients were treated with combination of transtuzumab, paclitaxel, carboplatin, and gemcitabine. The median number of chemotherapy

Table 3 Select active clinical trials for first-line therapy in advanced and metastatic bladder cancer ${ }^{100}$

\begin{tabular}{|c|c|c|c|c|}
\hline Study identifier & Phase & Study drug & Start date & Primary endpoint \\
\hline NCT0046I85I & II & gemcitabine + carboplatin + sorafenib & April 2007 & TTP \\
\hline NCTOII 26749 & $\mathrm{I} / \mathrm{II}$ & eribulin + gemcitabine + cisplatin & May 2010 & Safety \\
\hline NCT00635726 & II & $\begin{array}{l}\text { methotrexate }+ \text { vinblastine }+ \text { doxorubicin }+ \text { cisplatin followed } \\
\text { by gemcitabine }+ \text { cisplatin }\end{array}$ & March 2008 & ORR \\
\hline NCT00645593 & II & gemcitabine + cisplatin \pm cetuximab & March 2008 & ORR \\
\hline NCT00995488 & II & nab-paclitaxel + gemcitabine + carboplatin & October 2009 & Efficacy \\
\hline NCTOIII8039 & II & sunitinib (cisplatin ineligible patients) & May 2010 & TTP and safety \\
\hline NCTOII9I892 & II & gemcitabine + carboplatin \pm vandetanib & August 2009 & PFS \\
\hline NCT0I089088 & II & gemcitabine + cisplatin + sunitinib & March 2010 & PFS \\
\hline NCT0I090466 & $\mathrm{I} / \mathrm{II}$ & gemcitabine + cisplatin + temsirolimus & March 2010 & PFS and safety \\
\hline NCTOI2I5I36 & II & everolimus \pm paclitaxel (cisplatin ineligible patients) & October 2010 & ORR \\
\hline NCT00949455 & II/III & $\begin{array}{l}\text { lapatinib maintenance in HER-2+ bladder cancer after } \\
\text { first-line chemotherapy }\end{array}$ & July 2009 & PFS \\
\hline NCT00625664 & III & larotaxel + cisplatin vs gemcitabine + cisplatin (CILAB) & February 2008 & OS \\
\hline NCT0094233I & III & gemcitabine + cisplatin \pm bevacizumab & July 2009 & OS \\
\hline NCT00022191 & III & gemcitabine + cisplatin \pm paclitaxel & August 2001 & OS \\
\hline NCT00089I 28 & II & gemcitabine + irinotecan & August 2004 & ORR \\
\hline NCT0047836I & II & gemcitabine + paclitaxel + doxorubicin with pegfligrastim & May 2007 & ORR, TTP, and survival \\
\hline
\end{tabular}

Abbreviations: TTP, time to progression; ORR, objective response rate; OS, overall survival; PFS, progression-free survival. 
cycles administered was six. The ORR was an impressive 70\%; median TTP was 9.3 months and median OS was 14.1 months. Most common grade 3-4 toxicities were myelosuppression and sensory neuropathy; grade 3 cardiac toxicity was reported in two patients (4.5\%). Though the results are very promising, there appears no consensus on routinely screening for Her-2/neu expression on all bladder cancer specimens. Based on these results a prospective clinical Phase III study with paclitaxel, carboplatin, and gemcitabine, with or without trastuzumab, is clearly warranted.

\section{Second-line therapy}

An effective salvage therapy for relapsed urothelial cancer following first-line chemotherapy has remained an unmet need despite several research efforts. Frequently there is a significant deterioration in the overall clinical condition, often associated with renal impairment after progression following first-line therapy, which makes it difficult to enroll them in clinical trials, or even administer systemic chemotherapy off-study protocol. In several clinical trials, the reported response rates with single agents such as paclitaxel, ${ }^{79}$ ifosphamide, ${ }^{86}$ docetaxel,,${ }^{65}$ and gemcitabine ${ }^{64}$ has been about $20 \%$ or less. Combinations such as paclitaxel with gemcitabine, ${ }^{70,87}$ oxaliplatin with 5-fluorouracil (FOLFOX), ${ }^{88}$ or gemcitabine (GEM-OX) ${ }^{89}$ after failing M-VAC have demonstrated response rates in the range of $20 \%$ to $27 \%$ but with significant toxicities such as neutropenia, thrombocytopenia, and peripheral neuropathy. Currently, there is no defined standard second-line therapy for metastatic bladder cancer. Some of the more recent trials with (promising) results will be reviewed in this section.

Vinflunine is a novel, biflourinated, third-generation, vinca alkaloid, antimitotic agent that has demonstrated superior antitumor activity to other agents in its class. ${ }^{90}$ The efficacy of vinflunine as a second-line therapy for patients with relapsed or refractory advanced urothelial cancer after first-line platinum-containing chemotherapy has been evaluated in 3 open-label, multicenter studies. ${ }^{91-93}$ In the two Phase II studies, vinflunine demonstrated moderate antitumor activity with a RR of $15 \%{ }^{93}$ and $18 \% .^{92}$ The Phase III trial compared vinflunine plus best supportive care (BSC) with BSC alone. Patients (370) were randomly assigned in a 2:1 ratio to receive vinflunine plus BSC $(\mathrm{n}=253)$ or BSC alone $(n=117)$. Both arms were well balanced except there were more patients with performance status $>1$ (10\% difference) in the BSC arm. Most common grade $\geq 3$ toxicities for vinflunine arm were neutropenia (50\%), febrile neutropenia (6\%), anemia (19\%), fatigue (19\%), and constipation (16\%). In the intent-to-treat population, the objective of a median 2-month survival advantage (6.9 months for vinflunine plus BSC vs 4.6 months for BSC) was achieved but was not statistically significant $(P=0.287)$. Multivariate Cox analysis adjusting for prognostic factors showed a statistically significant effect of vinflunine on OS $(P=0.036)$, reducing the death risk by $23 \%$. ORR ( $8.6 \%$ vs $0 \%$ ), disease control $(41.4 \%$ vs $24.8 \%$ ), and PFS (3.0 vs 1.5 months) were all statistically significant, favoring vinflunine. With an acceptable safety profile, vinflunine appears to be a reasonable second-line therapy option for patients with bladder cancer who have relapsed following cisplatin-based therapy.

In a recent randomized Phase III trial by German Association of Urological Oncology (AB 20/99), ${ }^{94}$ shortterm (maximum of six cycles every 3 weeks) vs prolonged therapy (treatment continued until disease progression) with a combination of gemcitabine with paclitaxel was evaluated as second-line chemotherapeutic treatment for patients with metastatic urothelial cancer after failure of cisplatin-based first-line therapy. Of the 102 enrolled patients 96 were eligible for analysis. The results showed that there was no difference in OS (7.8 vs 8.0 months), PFS (4 vs 3.1 months), or ORR $(37.5 \%$ vs $41.5 \%)$ between the short-term and prolonged therapy. More patients had severe anemia (26\% vs $6.7 \%$ ) in the prolonged treatment arm. The high response rate $(\sim 40 \%)$ suggests that the combination of gemcitabine and paclitaxel is a reasonable option as second-line therapy in this group of patients.

Activity of single-agent pemetrexed as a second-line therapy in patients with urothelial cancer was reported by the Hoosier Oncology Group. ${ }^{95}$ Forty-seven patients were enrolled and included in the intention-to-treat efficacy analysis. The ORR was $27.7 \%$, median TTP was 2.9 months, median duration of response was 5 months, and median OS was 9.6 months, fatigue and myelosupression accounting for the most common grade 3-4 toxicity. This study supports pemetrexed as a reasonable second-line therapy option in this patient population.

Results of a Phase II study evaluating single-agent nab-paclitaxel, the albumin-bound nanoparticle formulation, in a cohort of 48 patients with urothelial cancer who had progressed or relapsed after cisplatin-based chemotherapy was recently presented. ORR in 47 evaluable patients was $32 \%$. With an additional $21 \%$ of patients having stable disease, the clinical benefit rate (CBR) was 53\%, representing one of the highest reported RRs in the second-line therapy of urothelial cancer. Nab-paclitaxel was well tolerated and the most frequent grade $\geq 3$ adverse events reported were pain (45\%), hypertension (14\%), and fatigue ( $8 \%)$. 
Ixabepilone, an epothilone B analog, which binds to $\beta$-tubulin and stabilizes microtubules, has shown promising activity in several solid tumors and is currently approved by FDA for treatment of metastatic breast cancer. ${ }^{96}$ Its efficacy in urothelial cancer was evaluated in a Phase II trial by ECOG (E3800). ${ }^{97}$ In this study of 45 patients, ORR was a dismal $11.9 \%$ with a median survival of 8 months. Toxicity was moderate, granulocytopenia, fatigue, and sensory neuropathy being the most common side-effects reported.

Signaling through VEGFR and EGFR pathways is thought to play a critical role in growth and progression of urothelial cancers. ${ }^{83}$ Several molecularly targeted approaches are currently under investigation as second-line therapies in recurrent/refractory bladder cancers (Table 4). A recent report of a multicenter, noncomparative randomized Phase II study ${ }^{98}$ of cetuximab with or without paclitaxel in patients with previously treated metastatic urothelial cancer suggests that EGFR inhibition with cetuximab enhances the antitumor activity of paclitaxel in this setting. Thirty-nine evaluable patients were enrolled. The cetuximab arm was closed after nine of the first eleven patients progressed by 8 weeks. ORR was $28.5 \%$, and median PFS for cetuximab-paclitaxel arm was 3.8 months with a median OS of 9.5 months. Pazopanib, a second-generation multitargeted tyrosine kinase inhibitor (TKi) of VEGFR-1, 2, and 3, platelet-derived growth factor receptor, and c-kit, has shown promising results as a single agent in an ongoing Phase II trial in heavily pretreated patients with relapsed or refractory urothelial cancer. ${ }^{99}$ In total, 18 patients were enrolled until July 2010, 10 patients having primary bladder tumor; $22 \%$ of patients had partial response and $61 \%$ had stable disease with a CBR of $83 \%$. The drug was well tolerated overall, with grade $\geq 3$ nausea or anorexia reported in two patients and hypertension in one patient. More patients need to be enrolled and longer follow-up is required. A Phase II study evaluating single-agent aflibercept, a soluble receptor for VEGF, also known as VEGF Trap, in urothelial cancer patients who have failed cisplatinum-based therapy has completed accrual but results are not yet reported. ${ }^{100}$ Clinical trials with other targeted agents such as lapatinib (HER-2 and EGFR TKi), erlotinib (HER-1 and HER-2 TKi), sunitinib (multiple receptor TKi), and everolimus (mTOR inhibitor) are ongoing (Table 4).

\section{Management of variants and nonurothelial cell malignancies of the bladder}

Primary nonurothelial bladder malignancies are rare, representing less than $10 \%$ of all bladder cancers. The recent World Health Organization classification of urothelial cancers lists 13 different histologic variants of urothelial cancer $^{101}$ (Table 5). The divergent differentiation patterns such as squamous, glandular (adenocarcinoma), micropapillary, nested, plasmacytoid, and carcinosarcoma/sarcomatoid variants should be identified because of the potential for having an unfavorable prognosis despite aggressive surgical management that relates both to an aggressive biological behavior and also often due to an advanced stage at the time of diagnosis.

Squamous cell carcinoma (SCC) is the second most prevalent epithelial neoplasm of the bladder, accounting for approximately $3 \%$ to $5 \%$ of bladder tumors in Western countries. ${ }^{102}$ While the pathogenesis of SCC of the bladder is only been partly understood, it is thought to involve factors that result in chronic bladder infection and irritation. SCC of the bladder in countries of the Middle East and Egypt has a distinct pathogenesis that is linked to chronic

Table 4 Select active clinical trials in second-line therapy for advanced and metastatic bladder cancer ${ }^{100}$

\begin{tabular}{lllll}
\hline Study identifier & Phase & Study drug & Start date & Primary endpoint \\
\hline NCT0I265940 & I/II & pazopanib + vinflunine & Dec 20I0 & PFS \\
NCT00365I57 & I/II & E7389 (halichondrin B analog) & October 20I0 & Response rate and safety \\
NCT00683059 & II & nab-paclitaxel & May 2008 & Response rate \\
NCT00578526 & II & sunitinib (SPRUCE) & December 2007 & PFS \\
NCT0I282463 & II & Docetaxel \pm ramucirumab or IMC-I8FI & January 20II & PFS \\
NCT0I2345I9 & I/II & AEZS-I08 in LHRH + urothelial cancers & November 2010 & MTD \\
NCT00722553 & II & pralatrexate & July 2008 & ORR \\
NCT0I03I875 & II & pazopanib & December 2009 & ORR \\
NCT0II08055 & II & pazopanib + paclitaxel & April 20I0 & ORR \\
NCT00933374 & II & everolimus + paclitaxel & July 2009 & ORR \\
\hline
\end{tabular}

Abbreviations: PFS, progression-free survival; MTD, maximum tolerated dose; ORR, objective response rate; AEZS-I08, luteinizing-hormone-releasing hormone linked to doxorubicin. 
Table 5 Variants of invasive urothelial carcinoma

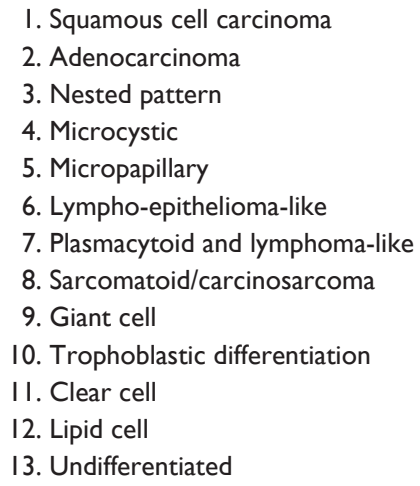

Note: Adapted from World Health Organization Classification of Tumours. IARC Press: Lyon; 2004:359. ${ }^{101}$

infections with Schistosoma haematobium. In regions where this water-borne parasitic pathogen is endemic, SCC not only represents the most common histological type of bladder tumor, but also the most prevalent form of cancer in men overall, accounting for $30 \%$ of cancers. Preoperative radiation has been shown to decrease pelvic recurrence in a single-institution study, but this remains of uncertain benefit. ${ }^{103}$ Standard chemotherapy regimens appear to have limited impact on the disease due to the relative chemoresistance of SCC. The use of chemotherapy regimens, such as the combination of paclitaxel, carboplatin, and gemcitabine, which have demonstrated efficacy in patients with SCC of other locations such as lung, head, and neck, may offer better outcomes. ${ }^{85}$ Standard treatment of Schistosoma-associated SCC is radical cystectomy and urinary diversion. A potential role for neoadjuvant or adjuvant radiation and chemotherapy remains poorly defined.

Pure adenocarcinoma of the bladder represents the third most common type of epithelial tumor comprising $0.5 \%$ to $2.0 \%$ of all bladder tumors. ${ }^{102}$ In advanced cases of adenocarcinoma of the bladder, conventional chemotherapy (eg, $\mathrm{M}-\mathrm{VAC}$ ) is not effective, and hence the use of chemotherapy or radiotherapy should be individualized and may be of potential benefit in select patients. A recent SEER-based analysis showed that while patients with adenocarcinoma of the bladder undergo radical cystectomy at more advanced disease stage, the stage- and grade-adjusted cancer-specific mortality is the same among patients with adenocarcinoma and urothelial carcinoma of the bladder. ${ }^{104}$ Primary small cell or neuroendocrine carcinoma of the bladder is an extremely uncommon, aggressive, poorly differentiated neoplasm that is similar to small cell carcinoma of the lung in clinical behavior and accounts for less than $0.7 \%$ of all bladder tumors. A report from Mayo Clinic suggest that more than half the patients had metastatic spread to the loco-regional lymph nodes, liver, or bone at the time of presentation. ${ }^{105}$ Chemotherapy regimens similar to those used in small cell lung cancer of the lung have been employed and shown to be of benefit in several retrospective studies. ${ }^{105,106}$

Sarcoma, a malignant mesenchymal tumor, and carcinosarcoma, a biphasic mixture of carcinoma and sarcoma, have very rare occurrence in the bladder with only a few case series reported to date. ${ }^{107-109}$ Metastatic sarcomas and carcinosarcomas are frequently treated by employing multimodality protocols including resection, radiation, and chemotherapy. Doxorubicin and ifosfamide appear to be the most active single agents. ${ }^{108} \mathrm{~A}$ case report suggested benefit using gemcitabine with cisplatin in a patient with metastatic sarcomatoid carcinoma. ${ }^{110}$

Overall, for patients with metastatic nonurothelial bladder cancers, patient management should be based upon the histology of the primary tumor. Given the absence of data showing a survival or quality-of-life benefit from chemotherapy for these diseases, palliative care as an alternative to chemotherapy should be offered. Those electing to receive chemotherapy should be encouraged to consider enrolling in a clinical trial if an appropriate trial is available.

\section{Concluding remarks}

Bladder cancer comprises a variety of diseases. While most patients with superficial cancers do not encounter a lifethreatening condition, several patients with invasive disease do. In this group of patients, choosing the appropriate systemic regimen and timing of institution of such therapy is crucial. Based on the review of the multiple randomized clinical trials and meta-analysis, the treatment paradigm for muscle-invasive bladder cancer has shifted from cystectomy alone towards the use of cisplatin-based neoadjuvant chemotherapy. Further, development of gene expression models (eg, 20-gene GEM) will allow patients who would benefit from such therapy to be identified more accurately. Understanding the biology and various pathways involved in development of invasive bladder cancer has led to evaluation of targeted therapy (eg, VEGFR and EGFR pathways and use of multityrosine TKi) in combination with conventional cytotoxic chemotherapy, and the results from such clinical trials are promising. Though the progress in the field of bladder cancer has been slow, the future looks bright. In view of the multitude of questions still unanswered, every patient with 
advanced bladder cancer should be encouraged to participate in clinical trials. Patients, physicians, and families should support each other in the quest to cure more patients and minimize mortality of bladder cancer.

\section{Disclosure}

The authors of this paper (Prakash Vishnu, Jacob Mathew, Winston W Tan) report no conflicts of interest.

\section{References}

1. Cancer Facts and Figures. American Cancer Society; 2010. http://www. cancer.org/acs/groups/content/@epidemiologysurveilance/documents/ document/acspc-026238.pdf. Accessed March 31, 2011.

2. SEER Stat Fact Sheets: bladder. National Cancer Institute; 2010. http:// seer.cancer.gov/statfacts/html/urinb.html. Accessed March 31, 2011.

3. Mostofi FK, Davis CJ, Sesterhenn IA. Pathology of Tumors of the Urinary Tract. Lyon, France: Saunders; 1988.

4. Shelley MD, Mason MD, Kynaston H. Intravesical therapy for superficial bladder cancer: a systematic review of randomised trials and meta-analyses. Cancer Treat Rev. 2010;36(3):195-205.

5. Soloway MS, Sofer M, Vaidya A. Contemporary management of stage T1 transitional cell carcinoma of the bladder. J Urol. 2002;167(4): 1573-1583.

6. Raghavan D, Shipley WU, Garnick MB, Russell PJ, Richie JP. Biology and management of bladder cancer. $N$ Engl J Med. 1990;322(16): 1129-1138.

7. Stein JP, Lieskovsky G, Cote R, et al. Radical cystectomy in the treatment of invasive bladder cancer: long-term results in 1,054 patients. J Clin Oncol. 2001;19(3):666-675.

8. Babaian RJ, Johnson DE, Llamas L, Ayala AG. Metastases from transitional cell carcinoma of urinary bladder. Urology. 1980;16(2): $142-144$.

9. Leissner J, Hohenfellner R, Thuroff JW, Wolf HK. Lymphadenectomy in patients with transitional cell carcinoma of the urinary bladder; significance for staging and prognosis. BJU Int. 2000;85(7):817-823.

10. AJCC Cancer Staging Manual. 7th ed. New York: springer; 2010.

11. Karl A, Carroll PR, Gschwend JE, et al. The impact of lymphadenectomy and lymph node metastasis on the outcomes of radical cystectomy for bladder cancer. Eur Urol. 2009;55(4):826-835.

12. Koppie TM, Vickers AJ, Vora K, Dalbagni G, Bochner BH. Standardization of pelvic lymphadenectomy performed at radical cystectomy: can we establish a minimum number of lymph nodes that should be removed? Cancer. 2006;107(10):2368-2374.

13. Stein JP, Skinner DG. The role of lymphadenectomy in high-grade invasive bladder cancer. Urol Clin North Am. 2005;32(2):187-197.

14. Fleischmann A, Thalmann GN, Markwalder R, Studer UE. Extracapsular extension of pelvic lymph node metastases from urothelial carcinoma of the bladder is an independent prognostic factor. $J$ Clin Oncol. 2005;23(10):2358-2365.

15. Loehrer PJ Sr, Einhorn LH, Elson PJ, et al. A randomized comparison of cisplatin alone or in combination with methotrexate, vinblastine, and doxorubicin in patients with metastatic urothelial carcinoma: a cooperative group study. J Clin Oncol. 1992;10(7):1066-1073.

16. Saxman SB, Propert KJ, Einhorn LH, et al. Long-term follow-up of a phase III intergroup study of cisplatin alone or in combination with methotrexate, vinblastine, and doxorubicin in patients with metastatic urothelial carcinoma: a cooperative group study. J Clin Oncol. 1997; 15(7):2564-2569.

17. von der Maase H, Sengelov L, Roberts JT, et al. Long-term survival results of a randomized trial comparing gemcitabine plus cisplatin, with methotrexate, vinblastine, doxorubicin, plus cisplatin in patients with bladder cancer. J Clin Oncol. 2005;23(21):4602-4608.
18. Lin CC, Hsu CH, Huang CY, et al. Prognostic factors for metastatic urothelial carcinoma treated with cisplatin and 5-fluorouracil-based regimens. Urology. 2007;69(3):479-484.

19. Karakiewicz PI, Shariat SF, Palapattu GS, et al. Precystectomy nomogram for prediction of advanced bladder cancer stage. Eur Urol. 2006;50(6):1254-1260; discussion 1261-1252.

20. Bochner BH, Kattan MW, Vora KC. Postoperative nomogram predicting risk of recurrence after radical cystectomy for bladder cancer. J Clin Oncol. 2006;24(24):3967-3972.

21. Shariat SF, Karakiewicz PI, Palapattu GS, et al. Nomograms provide improved accuracy for predicting survival after radical cystectomy. Clin Cancer Res. 2006;12(22):6663-6676.

22. Kuczyk MA, Bokemeyer C, Serth J, et al. p53 overexpression as a prognostic factor for advanced stage bladder cancer. Eur J Cancer. 1995;31 A(13-14):2243-2247.

23. Tsuji M, Kojima K, Murakami Y, Kanayama H, Kagawa S. Prognostic value of Ki-67 antigen and p53 protein in urinary bladder cancer: immunohistochemical analysis of radical cystectomy specimens. $\mathrm{Br} J$ Urol. 1997;79(3):367-372.

24. Lorenzo-Romero JG, Salinas-Sanchez AS, Gimenez-Bachs JM, et al. Prognostic implications of p53 gene mutations in bladder tumors. J Urol. 2003;169(2):492-499.

25. Sarkis AS, Bajorin DF, ReuterVE, et al. Prognostic value of p53 nuclear overexpression in patients with invasive bladder cancer treated with neoadjuvant MVAC. J Clin Oncol. 1995;13(6):1384-1390.

26. Hussain SA, Ganesan R, Hiller L, et al. Proapoptotic genes BAX and CD40L are predictors of survival in transitional cell carcinoma of the bladder. Br J Cancer. 2003;88(4):586-592.

27. Cooke PW, James ND, Ganesan R, Burton A, Young LS, Wallace DM. Bcl-2 expression identifies patients with advanced bladder cancer treated by radiotherapy who benefit from neoadjuvant chemotherapy. BJU Int . 2000;85(7):829-835.

28. Duggan B, Williamson K. Molecular markers for predicting recurrence, progression and outcomes of bladder cancer (do the poster boys need new posters?). Curr Opin Urol. 2004;14(5):277-286.

29. Takata R, Katagiri T, Kanehira M, et al. Predicting response to methotrexate, vinblastine, doxorubicin, and cisplatin neoadjuvant chemotherapy for bladder cancers through genome-wide gene expression profiling. Clin Cancer Res. 2005;11(7):2625-2636.

30. Hoffmann AC, Wild P, Leicht C, et al. MDR1 and ERCC1 expression predict outcome of patients with locally advanced bladder cancer receiving adjuvant chemotherapy. Neoplasia. 2010;12(8):628-636.

31. Bellmunt J, Paz-Ares L, Cuello M, et al. Gene expression of ERCC1 as a novel prognostic marker in advanced bladder cancer patients receiving cisplatin-based chemotherapy. Ann Oncol. 2007;18(3):522-528.

32. Takara K, Tsujimoto M, Kokufu M, Ohnishi N, Yokoyama T. Upregulation of MDR1 function and expression by cisplatin in LLCPK1 cells. Biol Pharm Bull. 2003;26(2):205-209.

33. Smith SC, Baras AS, Dancik G, et al. A 20-gene model for molecular nodal staging of bladder cancer: development and prospective assessment. Lancet Oncol. 2011;12(2):137-143.

34. Cookson MS, Herr HW, Zhang ZF, Soloway S, Sogani PC, Fair WR. The treated natural history of high risk superficial bladder cancer: 15-year outcome. J Urol. 1997;158(1):62-67.

35. Teramukai S, Nishiyama H, Matsui Y, Ogawa O, Fukushima M. Evaluation for surrogacy of end points by using data from observational studies: tumor downstaging for evaluating neoadjuvant chemotherapy in invasive bladder cancer. Clin Cancer Res. 2006;12(1): 139-143.

36. Neoadjuvant cisplatin, methotrexate, and vinblastine chemotherapy for muscle-invasive bladder cancer: a randomised controlled trial. International collaboration of trialists. Lancet. 1999;354(9178): 533-540.

37. Sherif A, Rintala E, Mestad O, et al. Neoadjuvant cisplatin-methotrexate chemotherapy for invasive bladder cancer - Nordic cystectomy trial 2. Scand J Urol Nephrol. 2002;36(6):419-425. 
38. Grossman HB, Natale RB, Tangen CM, et al. Neoadjuvant chemotherapy plus cystectomy compared with cystectomy alone for locally advanced bladder cancer. N Engl J Med. 2003;349(9):859-866.

39. Malmstrom PU, Rintala E, Wahlqvist R, Hellstrom P, Hellsten S, Hannisdal E. Five-year followup of a prospective trial of radical cystectomy and neoadjuvant chemotherapy: nordic cystectomy trial I. The Nordic Cooperative Bladder Cancer Study Group. J Urol. 1996;155(6):1903-1906.

40. Cortesi E. Neoadjuvant treatment for locally advanced bladder cancer: a randomized prospective clinical trial. American Society of Clinical Oncology Annual Meeting; 1995. Philadelphia, PA.

41. Vale CL; Meta-analysis Group MCTU, London, UK. Neoadjuvant chemotherapy in invasive bladder cancer: update of a systematic review and meta-analysis of individual patient data advanced bladder cancer (ABC) meta-analysis collaboration. Eur Urol. 2005;48(2):202-205; discussion 205-206.

42. Griffiths G, trialists. OboIco. International phase III trial assessing neoadjuvant cisplatin, methotrexate, and vinblastine chemotherapy for muscle-invasive bladder cancer: long-term results of the BA06 30894 Trial. J Clin Oncol. 2011. doi: 10.1200/JCO.2010.32.3139.

43. Herr HW, Faulkner JR, Grossman HB, et al. Surgical factors influence bladder cancer outcomes: a cooperative group report. J Clin Oncol. 2004;22(14):2781-2789.

44. Scosyrev E, Ely BW, Messing EM, et al. Do mixed histological features affect survival benefit from neoadjuvant platinum-based combination chemotherapy in patients with locally advanced bladder cancer? A secondary analysis of Southwest Oncology Group-Directed Intergroup Study (S8710). BJU Int. 2010. doi: 10.1111/j.1464-410X.2010.09900.x.

45. von der Maase H, Hansen SW, Roberts JT, et al. Gemcitabine and cisplatin versus methotrexate, vinblastine, doxorubicin, and cisplatin in advanced or metastatic bladder cancer: results of a large, randomized, multinational, multicenter, phase III study. J Clin Oncol. 2000;18(17):3068-3077.

46. Dash A, Pettus JA, Bochner BH, et al. Efficacy of neo-adjuvant gemcitabine plus cisplatin (GC) in muscle-invasive urothelial cancer (UC). American Society of Clinical Oncology Annual Meeting 2007; Chicago, IL.

47. Smith DC, Grivas P, Daignault S, et al. A phase II trial of neoadjuvant ABI-007, carboplatin, and gemcitabine (ACG) in patients with locally advanced carcinoma of the bladder. American Society of Clinical Oncology Genitourinary cancer symposium; 2011. Orlando, FL.

48. Blick C, Hall P, Pwint T, et al. Accelerated MVAC as neoadjuvant chemotherapy for patients with muscle-invasive transitional cell carcinoma of the bladder. American Society of Clinical Oncology Genitourinary Cancer Symposium; 2011. Orlando, FL, USA.

49. Rodel C, Grabenbauer GG, Kuhn R, et al. Combined-modality treatment and selective organ preservation in invasive bladder cancer: long-term results. J Clin Oncol. 2002;20(14):3061-3071.

50. Housset M, Dufour B, Durdux C, Chretien Y, Mejean A, Baillet F. Concurrent radio-chemotherapy in infiltrating cancer of the bladder: a new therapeutic approach? Cancer Radiother. 1998;2(Suppl 1): $67 \mathrm{~s}-72 \mathrm{~s}$.

51. Shipley WU, Winter KA, Kaufman DS, et al. Phase III trial of neoadjuvant chemotherapy in patients with invasive bladder cancer treated with selective bladder preservation by combined radiation therapy and chemotherapy: initial results of Radiation Therapy Oncology Group 89-03. J Clin Oncol. 1998;16(11):3576-3583.

52. Kachnic LA, Kaufman DS, Heney NM, et al. Bladder preservation by combined modality therapy for invasive bladder cancer. J Clin Oncol. 1997;15(3):1022-1029.

53. Herr HW, Bajorin DF, Scher HI. Neoadjuvant chemotherapy and bladder-sparing surgery for invasive bladder cancer: ten-year outcome. J Clin Oncol. 1998;16(4):1298-1301.

54. Sternberg CN, Pansadoro V, Calabro F, et al. Can patient selection for bladder preservation be based on response to chemotherapy? Cancer. 2003;97(7):1644-1652.
55. DeVere White RW, Lara PN Jr, Goldman B, et al. A sequential treatment approach to myoinvasive urothelial cancer: a phase II Southwest Oncology Group trial (S0219). J Urol. 2009;181(6):2476-2480; discussion 2480-2471.

56. Calabro F, Sternberg CN. Neoadjuvant and adjuvant chemotherapy in muscle-invasive bladder cancer. Eur Urol. 2009;55(2):348-358.

57. Vale CL, On behalf of Meta-analysis Group MCTU, London, UK. Adjuvant chemotherapy in invasive bladder cancer: a systematic review and meta-analysis of individual patient data Advanced Bladder Cancer (ABC) Meta-analysis Collaboration. Eur Urol. 2005;48(2):189-199; discussion 199-201.

58. Cognetti F, Ruggeri EM, Felici A, et al. Adjuvant chemotherapy (AC) with cisplatin + gemcitabine $(\mathrm{CG})$ versus chemotherapy $(\mathrm{CT})$ at relapse (CR) in patients (pts) with muscle-invasive bladder cancer (MIBC) submitted to radical cystectomy (RC). An Italian multicenter randomised phase III trial. American Society of Clinical Oncology Annual Meeting; 2008. Chicago, IL

59. Stadler WM, Lerner SP, Groshen S, et al. Randomized trial of p53 targeted adjuvant therapy for patients (pts) with organ- confined nodenegative urothelial bladder cancer (UBC). American Society of Clinical Oncology Annual Meeting; 2009. Chicago, IL.

60. Paz-Ares G, Solsona E, Esteban E, et al. Randomized phase III trial comparing adjuvant paclitaxel/gemcitabine/cisplatin (PGC) to observation in patients with resected invasive bladder cancer: results of the Spanish Oncology Genitourinary Group (SOGUG) 99/01study. American Society of Clinical Oncology Annual Meeting; 2010. Chicago, IL.

61. Meyers FJ, Palmer JM, Freiha FS, et al. The fate of the bladder in patients with metastatic bladder cancer treated with cisplatin, methotrexate and vinblastine: a northern california oncology group study. J Urol. 1985;134(6):1118-1121.

62. Oliver RT, England HR, Risdon RA, Blandy JP. Methotrexate in the treatment of metastatic and recurrent primary transitional cell carcinoma. J Urol. 1984;131(3):483-485.

63. Soloway MS, Einstein A, Corder MP, Bonney W, Prout GR Jr, Coombs J. A comparison of cisplatin and the combination of cisplatin and cyclophosphamide in advanced urothelial cancer. A National Bladder Cancer Collaborative Group A Study. Cancer. 1983;52(5): 767-772.

64. Lorusso V, Pollera CF, Antimi M, et al. A phase II study of gemcitabine in patients with transitional cell carcinoma of the urinary tract previously treated with platinum. Italian Co-operative Group on Bladder Cancer. Eur J Cancer. 1998;34(8):1208-1212.

65. McCaffrey JA, Hilton S, Mazumdar M, et al. Phase II trial of docetaxel in patients with advanced or metastatic transitional-cell carcinoma. J Clin Oncol. 1997;15(5):1853-1857.

66. Pronzato P, Vigani A, Pensa F, Vanoli M, Tani F, Vaira F. Second line chemotherapy with ifosfamide as outpatient treatment for advanced bladder cancer. Am J Clin Oncol. 1997;20(5):519-521.

67. Hillcoat BL, Raghavan D, Matthews J, et al. A randomized trial of cisplatin versus cisplatin plus methotrexate in advanced cancer of the urothelial tract. J Clin Oncol. 1989;7(6):706-709.

68. Sternberg CN, Yagoda A, Scher HI, et al. Methotrexate, vinblastine, doxorubicin, and cisplatin for advanced transitional cell carcinoma of the urothelium. Efficacy and patterns of response and relapse. Cancer. 1989;64(12):2448-2458.

69. Logothetis CJ, Dexeus FH, Finn L, et al. A prospective randomized trial comparing MVAC and CISCA chemotherapy for patients with metastatic urothelial tumors. J Clin Oncol. 1990;8(6):1050-1055.

70. Sternberg CN, de Mulder PH, Schornagel JH, et al. Randomized phase III trial of high-dose-intensity methotrexate, vinblastine, doxorubicin, and cisplatin (MVAC) chemotherapy and recombinant human granulocyte colony-stimulating factor versus classic MVAC in advanced urothelial tract tumors: European Organization for Research and Treatment of Cancer Protocol no. 30924. J Clin Oncol. 2001;19(10): 2638-2646. 
71. Sternberg CN, de Mulder P, Schornagel JH, et al. Seven year update of an EORTC phase III trial of high-dose intensity M-VAC chemotherapy and G-CSF versus classic M-VAC in advanced urothelial tract tumours. Eur J Cancer. 2006;42(1):50-54.

72. von der Maase H, Andersen L, Crino L, Weinknecht S, Dogliotti L. Weekly gemcitabine and cisplatin combination therapy in patients with transitional cell carcinoma of the urothelium: a phase II clinical trial. Ann Oncol. 1999;10(12):1461-1465.

73. Adamo V, Magno C, Spitaleri G, et al. Phase II study of gemcitabine and cisplatin in patients with advanced or metastatic bladder cancer: long-term follow-up of a 3-week regimen. Oncology. 2005;69(5): 391-398.

74. Bellmunt J, von der Maase H, Mead GM, et al. Randomized phase III study comparing paclitaxel/cisplatin/gemcitabine (PCG) and gemcitabine/cisplatin (GC) in patients with locally advanced (LA) or metastatic (M) urothelial cancer without prior systemic therapy. American Society of Clinical Oncology Annual Meeting2007; Chicago, IL.

75. Bamias A, Aravantinos G, Deliveliotis C, et al. Docetaxel and cisplatin with granulocyte colony-stimulating factor (G-CSF) versus MVAC with G-CSF in advanced urothelial carcinoma: a multicenter, randomized, phase III study from the Hellenic Cooperative Oncology Group. J Clin Oncol. 2004;22(2):220-228.

76. Dogliotti L, Carteni G, Siena S, et al. Gemcitabine plus cisplatin versus gemcitabine plus carboplatin as first-line chemotherapy in advanced transitional cell carcinoma of the urothelium: results of a randomized phase 2 trial. Eur Urol. 2007;52(1):134-141.

77. Dreicer R, Manola J, Roth BJ, et al. Phase III trial of methotrexate, vinblastine, doxorubicin, and cisplatin versus carboplatin and paclitaxel in patients with advanced carcinoma of the urothelium. Cancer. 2004;100(8):1639-1645.

78. De Santis M, Bellmunt J, Mead G, et al. Randomized phase II/III trial assessing gemcitabine/ carboplatin and methotrexate/carboplatin/vinblastine in patients with advanced urothelial cancer "unfit" for cisplatinbased chemotherapy: phase II - results of EORTC study 30986. J Clin Oncol. 2009;27(33):5634-5639.

79. Dreicer R, Li H, Cooney MM, Wilding G, Roth BJ. Phase 2 trial of pemetrexed disodium and gemcitabine in advanced urothelial cancer (E4802): a trial of the Eastern Cooperative Oncology Group. Cancer. 2008;112(12):2671-2675.

80. von der Maase H, Lehmann J, Gravis G, et al. A phase II trial of pemetrexed plus gemcitabine in locally advanced and/or metastatic transitional cell carcinoma of the urothelium. Ann Oncol. 2006;17(10): $1533-1538$

81. Cortes J, O'Shaughnessy J, Loesch D, et al. Eribulin monotherapy versus treatment of physician's choice in patients with metastatic breast cancer (EMBRACE): a phase 3 open-label randomised study. Lancet. 2011;377(9769):914-923.

82. Quinn D, I., Aparicio A, Tsao-Wei DD, et al. Phase II study of eribulin (E7389) in patients (pts) with advanced urothelial cancer (UC) - Final report: a California Cancer Consortium-led NCI/CTEP-sponsored trial. American Society of Clinical Oncology Annual Meeting; 2010. Chicago, IL.

83. Mitra AP, Lin H, Datar RH, Cote RJ. Molecular biology of bladder cancer: prognostic and clinical implications. Clin Genitourin Cancer. 2006;5(1):67-77.

84. Hahn NM, Stadler WM, Zon R, et al. Mature results from Hoosier Oncology Group GU04-75 phase II trial of cisplatin (C), gemcitabine (G), and bevacizumab (B) as first-line chemotherapy for metastatic urothelial carcinoma (UC). American Society of Clinical Oncology Annual Meeting; 2010. Chicago, IL.

85. Hussain M, Vaishampayan U, Du W, Redman B, Smith DC. Combination paclitaxel, carboplatin, and gemcitabine is an active treatment for advanced urothelial cancer. J Clin Oncol. 2001;19(9):2527-2533.

86. Witte RS, Elson P, Bono B, et al. Eastern Cooperative Oncology Group phase II trial of ifosfamide in the treatment of previously treated advanced urothelial carcinoma. J Clin Oncol. 1997;15(2):589-593.
87. Meluch AA, Greco FA, Burris HA 3rd, et al. Paclitaxel and gemcitabine chemotherapy for advanced transitional-cell carcinoma of the urothelial tract: a phase II trial of the Minnie pearl cancer research network. J Clin Oncol. 2001;19(12):3018-3024.

88. Di Lorenzo G, Autorino R, Giordano A, et al. FOLFOX-4 in pre-treated patients with advanced transitional cell carcinoma of the bladder. Jpn $J$ Clin Oncol. 2004;34(12):747-750.

89. Culine S, Rebillard X, Iborra F, et al. Gemcitabine and oxaliplatin in advanced transitional cell carcinoma of the urothelium: a pilot study. Anticancer Res. 2003;23(2C):1903-1906.

90. Kruczynski A, Hill BT. Vinflunine, the latest Vinca alkaloid in clinical development. A review of its preclinical anticancer properties. Crit Rev Oncol Hematol. 2001;40(2):159-173.

91. Bellmunt J, Theodore C, Demkov T, et al. Phase III trial of vinflunine plus best supportive care compared with best supportive care alone after a platinum-containing regimen in patients with advanced transitional cell carcinoma of the urothelial tract. J Clin Oncol. 2009;27(27):4454-4461.

92. Culine S, Theodore C, De Santis M, et al. A phase II study of vinflunine in bladder cancer patients progressing after first-line platinumcontaining regimen. Br J Cancer. 2006;94(10):1395-1401.

93. Vaughn DJ, Srinivas S, Stadler WM, et al. Vinflunine in platinumpretreated patients with locally advanced or metastatic urothelial carcinoma: results of a large phase 2 study. Cancer. 2009;115(18): 4110-4117.

94. Albers P, Park SI, Niegisch G, et al. Randomized phase III trial of 2nd line gemcitabine and paclitaxel chemotherapy in patients with advanced bladder cancer: short-term versus prolonged treatment (German Association of Urological Oncology (AUO) trial AB 20/99). Ann Oncol. 2011;22(2):288-294.

95. Sweeney CJ, Roth BJ, Kabbinavar FF, et al. Phase II study of pemetrexed for second-line treatment of transitional cell cancer of the urothelium. J Clin Oncol. 2006;24(21):3451-3457.

96. Toppmeyer DL, Goodin S. Ixabepilone, a new treatment option for metastatic breast cancer. Am J Clin Oncol. 2010;33(5):516-521.

97. Dreicer R, Li S, Manola J, Haas NB, Roth BJ, Wilding G. Phase 2 trial of epothilone B analog BMS-247550 (ixabepilone) in advanced carcinoma of the urothelium (E3800): a trial of the Eastern Cooperative Oncology Group. Cancer. 2007;110(4):759-763.

98. Wong Y, Litwin S, Plimack ER, et al. Effect of EGFR inhibition with cetuximab on the efficacy of paclitaxel in previously treated metastatic urothelial cancer. American Society of Clinical Oncology Genitourinary Cancers Symposium; 2011. Orlando, FL.

99. Necchi A, Nicolai N, Guglielmi A, et al. Phase II study of pazopanib monotherapy for patients with relapsed/refractory urothelial cancer 35th Europenan Society of Medical Oncology Congress; 2010. Milan, Italy.

100. Availabe from: http://www.Clinicaltrials.gov. 2010. Accessed March 31, 2011.

101. Eble JN, Sauter G, Epstein JI, Sesterhenn IA. World Health Organization Classification of Tumours. World Health Organization Classification of Tumours. Lyon, France: IARC Press; 2004:359.

102. Dahm P, Gschwend JE. Malignant non-urothelial neoplasms of the urinary bladder: a review. Eur Urol. 2003;44(6):672-681.

103. Swanson DA, Liles A, Zagars GK. Preoperative irradiation and radical cystectomy for stages T2 and T3 squamous cell carcinoma of the bladder. J Urol. 1990;143(1):37-40.

104. Lughezzani G, Sun M, Jeldres C, et al. Adenocarcinoma versus urothelial carcinoma of the urinary bladder: comparison between pathologic stage at radical cystectomy and cancer-specific mortality. Urology. 2010;75(2):376-381.

105. Choong NW, Quevedo JF, Kaur JS. Small cell carcinoma of the urinary bladder. The Mayo Clinic experience. Cancer. 2005;103(6):1172-1178.

106. Bex A, Nieuwenhuijzen JA, Kerst M, et al. Small cell carcinoma of bladder: a single-center prospective study of 25 cases treated in analogy to small cell lung cancer. Urology. 2005;65(2):295-299. 
107. Spiess PE, Kassouf W, Steinberg JR, et al. Review of the M.D. Anderson experience in the treatment of bladder sarcoma. Urol Oncol. 2007;25(1):38-45.

108. Russo P, Brady MS, Conlon K, et al. Adult urological sarcoma. J Urol. 1992;147(4):1032-1036; discussion 1036-1037.

109. Lopez-Beltran A, Pacelli A, Rothenberg HJ, et al. Carcinosarcoma and sarcomatoid carcinoma of the bladder: clinicopathological study of 41 cases. J Urol. 1998;159(5):1497-1503.
110. Froehner M, Gaertner HJ, Manseck A, Wirth MP. Durable complete remission of metastatic sarcomatoid carcinoma of the bladder with cisplatin and gemcitabine in an 80-year-old man. Urology. 2001;58(5):799.

\section{Publish your work in this journal}

OncoTargets and Therapy is an international, peer-reviewed, open access journal focusing on the pathological basis of all cancers, potential targets for therapy and treatment protocols employed to improve the management of cancer patients. The journal also focuses on the impact of management programs and new therapeutic agents and protocols on

\section{Dovepress}

patient perspectives such as quality of life, adherence and satisfaction. The manuscript management system is completely online and includes a very quick and fair peer-review system, which is all easy to use. Visit http://www.dovepress.com/testimonials.php to read real quotes from published authors.

Submit your manuscript here: http://www.dovepress.com/oncotargets-and-therapy-journal 